\title{
Mobility and climate protection - friends or enemies?
}

Thorsten Herdan,

Federal Ministry for Economic Affairs and Energy (BMWi)

Die Unterlagen wurden nicht zur Veröffentlichung freigegeben. Wir bitten um Verständnis. 